\title{
Basal cell carcinoma arising within a seborrheic keratosis with respect to immunohistochemical characteristics
}

\author{
MITSUAKI ISHIDA $^{1}$, NORIKO OHSATO ${ }^{2}$ and HIDETOSHI OKABE ${ }^{1}$ \\ ${ }^{1}$ Department of Clinical Laboratory Medicine and Division of Diagnostic Pathology, Shiga University \\ of Medical Science, Otsu, Shiga 520-2192; ${ }^{2}$ Ohsato Dermatological Clinic, Kobe, Hyogo 651-1245, Japan
}

Received March 28, 2011; Accepted April 29, 2011

DOI: $10.3892 / \mathrm{ol} .2011 .303$

\begin{abstract}
Malignant tumor occurring within seborrheic keratosis (SK), which is one of the most common benign cutaneous tumors, is extremely rare. We report a case of basal cell carcinoma (BCC) arising within SK. Additionally, this is the first study to describe the immunohistochemical characteristics of this type of carcinoma. An 89-year-old Japanese woman presented with a persistent scaly plaque in the right auricle of her ear. Histopathological study revealed a superficial type of BCC arising within SK. Immunohistochemical studies showed that cytokeratin 17 (CK17), CK19, SOX9 and p53 protein were expressed in BCC, but not in SK. BCC is considered to originate from the follicular germinative cells, and the outer root sheath may be the possible origin. SK is also thought to be a benign skin appendage neoplasm showing follicular differentiation, especially follicular infundibula. Therefore, previous reports speculated that there was a pathogenic relationship between SK and BCC, with respect to a common follicular origin. However, the immunohistochemical characteristics of this study suggest that BCC does not arise directly from SK, but instead, that SK is the nidus of the carcinoma, resulting in the abutment of SK with BCC. Furthermore, the results of the present case suggest that immunohistochemical surveillance of the expression of CK17, CK19 and SOX9 and p53 protein is useful in differentiating minute $\mathrm{BCC}$ from the non-neoplastic hair buds.
\end{abstract}

\section{Introduction}

Seborrheic keratosis (SK) is one of the most common benign cutaneous tumors encountered in the daily dermatopathological practice. Malignant tumor occurring within SK is extremely rare; one retrospective study reported that only $0.6 \%$ of SK showed direct contiguity with malignant tumors (1).

Correspondence to: Dr Mitsuaki Ishida, Department of Clinical Laboratory Medicine and Division of Diagnostic Pathology, Shiga University of Medical Science, Tsukinowa-cho, Seta, Otsu, Shiga 520-2192, Japan

E-mail: mitsuaki@belle.shiga-med.ac.jp

Key words: basal cell carcinoma, seborrheic keratosis, SOX9
Basal cell carcinoma (BCC) arising within SK is examined in this pioneering study, which also describes the immunohistochemical characteristics of this type of lesion.

\section{Case Report}

An 89-year-old Japanese woman presented with a persistent scaly plaque, measuring $14 \times 5 \mathrm{~mm}$, in the right auricle of her ear. Biopsy of the lesion was performed, under the clinical diagnosis of SK. After the pathological diagnosis of the biopsy specimen, the patient underwent complete tumor resection.

\section{Materials and methods}

The formalin-fixed, paraffin-embedded tissue blocks were cut into 3- $\mu \mathrm{m}$ sections, deparaffinized and rehydrated. Each section was stained with hematoxylin and eosin, and used for immunohistochemical analyses. Immunohistochemical analyses were performed using an autostainer (XT system Benchmark, Ventana Medical System, Tucson, AZ, USA) according to the manufacturer's instructions. The primary antibodies used were: a mouse monoclonal antibody against cytokeratin (CK17) (E3, Novocastra Laboratories, Ltd., Newcastle upon Tyne, UK), a mouse monoclonal antibody against CK19 (clone b170, Novocastra Laboratories, Newcastle, UK), a mouse monoclonal antibody against p53 (PAb1801; Novocastra Laboratories), and a rabbit polyclonal antibody against SOX9 (Santa Cruz Biotechnology, Santa Cruz, CA, USA).

\section{Results}

Histopathological study of the biopsy specimen revealed hyperkeratosis and proliferation of basaloid and squamoid cells in the acanthotic epidermis. These basaloid and squamoid cells were without atypia, and mitotic figures were rarely noted (Fig. 1). These were typical histopathological findings for SK. There were some buds composed of follicular germinative cell-like immature basaloid cells directly attached to the undersurface of the SK (Fig. 1). These immature basaloid cells had large elongated nuclei and scant cytoplasm, and showed peripheral palisading (Fig. 1, inset). Mitotic figures and apoptotic bodies were scattered. There were areas of retraction of the stroma around the tumor buds. These 


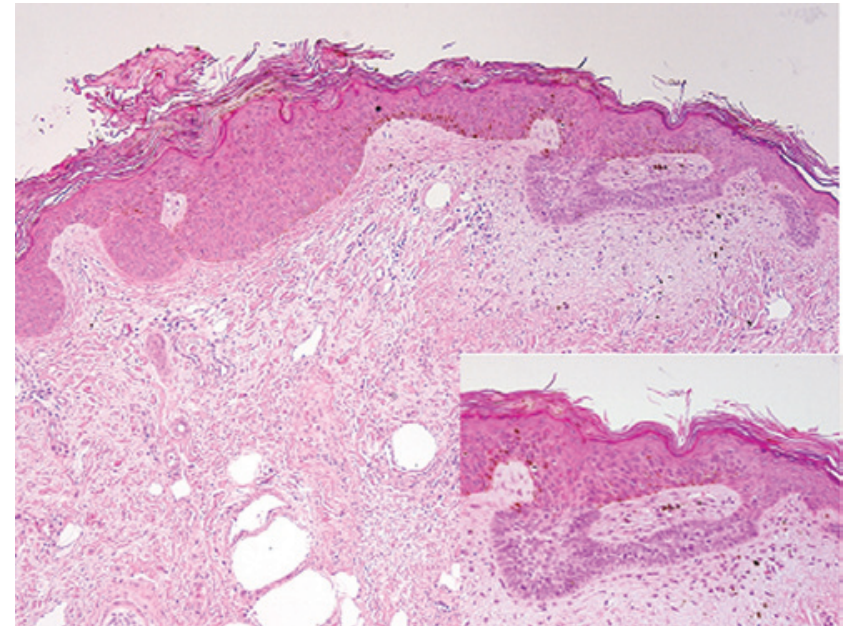

Figure 1. Histopathological findings of a tumor found in the ear auricle Epithelial buds composed of follicular germinative cell-like immature basaloid cells directly attached to the undersurface of seborrheic keratosis (H\&E stain). Original magnification, x100 (inset, x200).

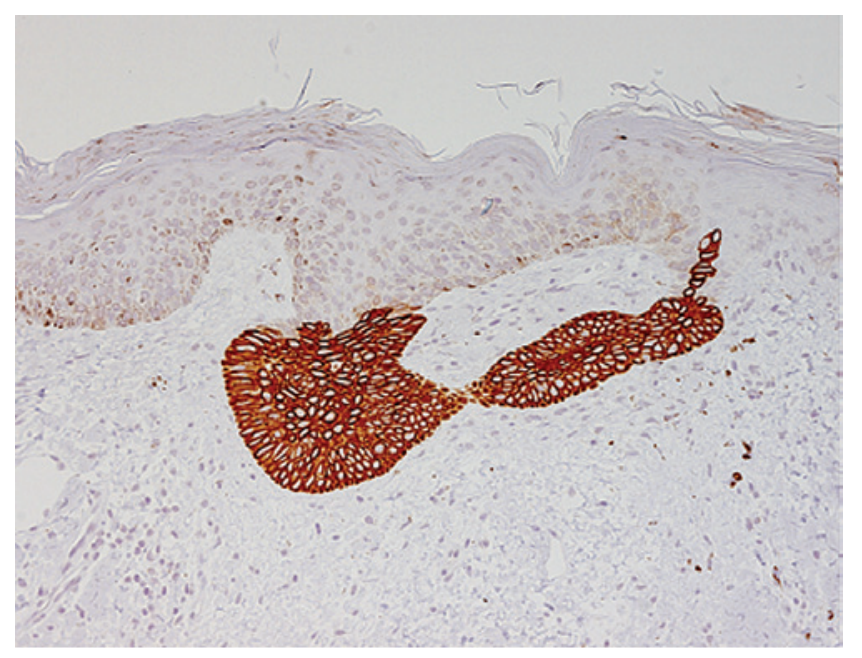

Figure 2. Immunohistochemically, CK 17 was expressed in the basal cell carcinoma, but not in the seborrheic keratosis. Original magnification, x200.

histopathological findings corresponded to the superficial type of BCC. Therefore, a diagnosis of the superficial type of BCC arising within SK was ultimately made based on the biopsy specimen.

Immunohistochemical studies revealed that CK17, CK19 and SOX9 were expressed in the BCC, but not in the SK (Fig. 2). Furthermore, the overexpression of p53 protein was observed in the BCC, but not in the SK (Fig. 3).

The histopathological and immunohistochemical findings of the surgically resected specimen were identical to those of the biopsy specimen.

\section{Discussion}

The development of a malignant neoplasm in association with SK was previously identified. BCC, squamous cell carcinoma and malignant melanoma have been documented to occur within SK, and BCC is the most common malignant neoplasm occurring within SK (1,2). The predominant histological

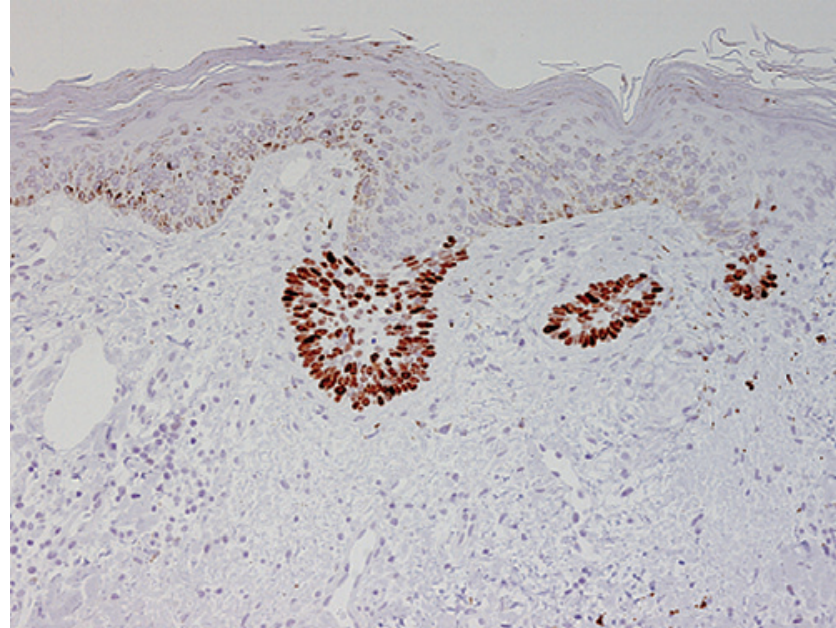

Figure 3. Overexpression of p53 protein was observed in the basal cell carcinoma, but not in the seborrheic keratosis. Original magnification, x200.

subtype of BCC associated with SK is the superficial type, as observed in the present case (2).

This report is the first to describe the immunohistochemical characteristics of BCC arising within SK. We found that CK17, CK19 and SOX9 were expressed in the BCC, but not in the SK. Findings of previous reports revealed that CK17 and CK19 were expressed in the outer root sheath of hair follicles as well as in most cases of BCC (3), but not in SK (4). SOX9 belongs to the SOX gene family, members of which are known to be involved in a large number of developmental processes. Recent studies showed that SOX 9 was expressed in the outer root sheath of hair follicles, sweat and sebaceous glands, as well as BCC $(5,6)$; however, its expression was rarely observed in SK (6). Thus, the immunohistochemical findings of the present case are typical for SK and $\mathrm{BCC}$.

$\mathrm{BCC}$ is considered to originate from follicular germinative cells, and various immunohistochemical studies have indicated that the outer root sheath of hair follicles may be a possible origin of BCC (3). In addition, SK is also thought to be a benign skin appendage neoplasm showing follicular differentiation, especially follicular infundibula. Therefore, Cascajo et al speculated that there was a pathogenic relationship between SK and BCC, with respect to a common follicular origin (2). However, the immunohistochemical characteristics of BCC and SK in the present case were different, suggesting that BCC does not arise directly from SK, but instead, that SK is the nidus of the carcinoma, resulting in the abutment of SK with BCC.

Furthermore, the results of the present case suggest that immunohistochemical surveillance of the expression of CK17, CK19, SOX 9, and p53 protein is useful in differentiating minute $\mathrm{BCC}$, especially the superficial type, from the nonneoplastic hair buds, as it is not always easy to distinguish between them.

\section{References}

1. LimC: Seborrheic keratoses with associated lesions: aretrospective analysis of 85 lesions. Australas J Dermatol 47: 109-113, 2006. 
2. Cascajo CD, Reichel M and Sanchez JL: Malignant neoplasms associated with seborrheic keratosis. An analysis of 54 cases. Am J Dermatopathol 18: 278-282, 1996.

3. Ishida M, Kushima $\mathrm{R}$ and Okabe $\mathrm{H}$ : Immunohistochemical demonstration of D2-40 in basal cell carcinomas of the skin. J Cutan Pathol 35: 926-930, 2008.

4. Liu HN, Chang YT and Chen CC: Differential of hidroacanthoma simplex from clonal seborrheic keratosis - an immunohistochemical study. Am J Dermatopathol 26: 188-193, 2004.
5. Krahl D and Sellheyer K: Sox9, more than a marker of the outer root sheath: spatiotemporal expression pattern during human cutaneous embyogenesis. J Cutan Pathol 37: 350-356, 2010.

6. Vidal VPI, Ortonne N and Schedl A: SOX9 expression is a general marker of basal cell carcinoma and adnexal-related neoplasms. J Cutan Pathol 35: 373-379, 2008. 